\title{
Stat3 modulates chloride channel accessory protein expression in normal and neoplastic mammary tissue
}

\author{
Katherine Hughes ${ }^{*, 1}$, Maximilian Blanck², Sara Pensa ${ }^{2,3}$ and Christine J Watson ${ }^{2}$
}

Mammary gland regression at the cessation of lactation (involution) is an exquisitely orchestrated process of cell death and tissue remodelling in which Stat3 signalling has an essential role. The involution microenvironment of the mammary gland is considered to be pro-tumourigenic and a proportion of cases of pregnancy-associated breast cancer are suggested to originate in tandem with involution. However, the apparent paradox that STAT3 is required for cell death in normal mammary gland, but is associated with breast cancer cell survival, has not been resolved. Herein, we investigate Stat3-mediated regulation of expression of members of the calcium-activated chloride channel regulator (CLCA) family of proteins during involution and mammary carcinogenesis. Using the conditionally immortal mammary epithelial cell line KIM-2, together with mice exhibiting mammary epithelial cell-specific deletion of Stat3 during lactation, we demonstrate that expression of mCLCA1 and mCLCA2 is elevated in concert with activation of Stat3. By contrast, murine CLCA5 (mCLCA5), the murine orthologue of human CLCA2, is significantly upregulated at 24, 72 and $96 \mathrm{~h}$ of involution in Stat3 knockout mice, suggesting a reciprocal regulation of these proteins by Stat3 in vivo. Interestingly, orthotopic tumours arising from transplantation of 4T1 murine mammary tumour cells exhibit both phosphorylated Stat3 and mCLCA5 expression. However, we demonstrate that expression is highly compartmentalized to distinct subpopulations of cells, and that Stat3 retains a suppressive effect on $\mathrm{mCLCA} 5$ expression in 4T1 tumour cells. These findings enhance our understanding of the regulation of CLCA channel expression both in vitro and in vivo, and in particular, demonstrate that expression of mCLCA1 and $\mathrm{mCLCA} 2$ during involution is profoundly dependent upon Stat3, whereas the relationship between mCLCA5 and Stat3 activity is reciprocal and restricted to different subpopulations of cells.

Cell Death and Disease (2016) 7, e2398; doi:10.1038/cddis.2016.302; published online 6 October 2016

At the cessation of lactation, the two-phase process of mammary gland involution restores the organ to near its prepregnant state, and is exquisitely regulated by factors including transforming growth factor- $\beta 3$ during the first phase of involution, ${ }^{1}$ IL-6 cytokine family members leukaemia inhibitory factor (LIF) (first phase) ${ }^{2,3}$ and oncostatin M (OSM) (second phase). ${ }^{4}$ These cytokines activate Stat3, a critical transcriptional coordinator of the involution process. ${ }^{5,6}$ Thus, mice with a mammary-specific conditional deletion of Stat3 exhibit markedly impaired involution and diminished cell death. $^{7-10}$

Although the initial phase of involution is reversible, the second irreversible stage incorporates further cell death, extensive tissue remodelling and acquisition of a 'woundhealing phenotype'. ${ }^{11}$ The inflammatory milieu of the involution mammary gland is considered to be pro-tumourigenic, illustrated by mouse models of involution-associated tumourigenesis. ${ }^{12,13} \mathrm{~A}$ corollary of these observations may be found in epidemiological evidence from human breast cancer indicating not only that a subgroup of cases of the wider set of 'pregnancy-associated breast cancers' arises in the post-partum period, but that this subset of patients may have a poorer prognosis. ${ }^{14}$

Chloride channel accessory proteins, also known as calcium-activated chloride channel regulators (CLCA proteins), are a family of transmembrane proteins that have been suggested to have a role in chloride conductance in epithelial cells. Although the mechanism by which they activate other channel proteins to trigger movement of chloride ions across membranes is not clear, there is some evidence that they can be secreted, and thereby potentially activate chloride channels. $^{15}$

CLCA proteins have been associated with diverse functions including cell adhesion, ${ }^{16}$ apoptosis, tumourigenesis and mucus cell differentiation. ${ }^{17}$ Of particular interest, both CLCA2 and CLCA4 have been shown to inhibit proliferation of breast cancer cells when ectopically expressed in vitro, ${ }^{18}$ whereas normal breast tissue exhibits high levels of CLCA2 in both acini and small ducts. ${ }^{19}$ Murine CLCA5 (mCLCA5) is highly homologous to human CLCA2 (hCLCA2) and is considered to be its orthologue. ${ }^{20}$ Widely expressed in the cytoplasmic granules of granular layer keratinocytes in stratified squamous epithelia, ${ }^{21}$ mCLCA5 is downregulated in some murine

\footnotetext{
${ }^{1}$ Department of Veterinary Medicine, University of Cambridge, Madingley Road, Cambridge CB3 OES, UK and ${ }^{2}$ Department of Pathology, University of Cambridge, Tennis Court Road, Cambridge CB2 1QP, UK

${ }^{*}$ Corresponding author: K Hughes, Department of Veterinary Medicine, University of Cambridge, Madingley Road, Cambridge CB3 0ES, UK. Tel: +44 1223 337607 ; Fax: +44 1223 337605; E-mail: kh387@cam.ac.uk

${ }^{3}$ Current address: Department of Pharmacology, University of Cambridge, Tennis Court Road, Cambridge CB2 1PD, UK.

Abbreviations: CLCA, chloride channel regulator, calcium-activated, also known as chloride channel accessory protein; LIF, leukaemia inhibitory factor; OSM, oncostatin M; Stat3, signal transducer and activator of transcription 3

Received 17.5.16; revised 14.8.16; accepted 22.8.16; Edited by A Stephanou
} 
a
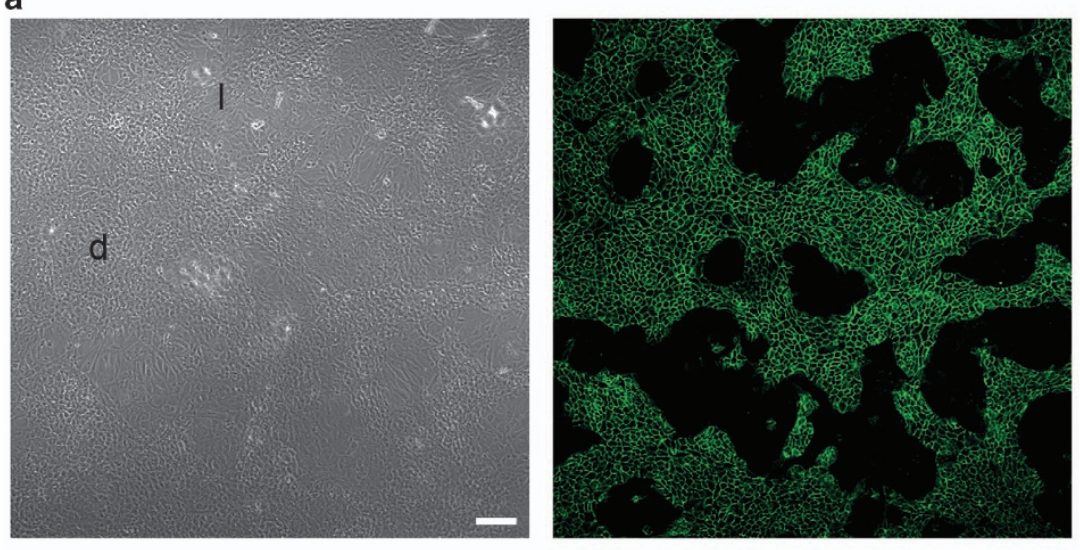

E-cadherin

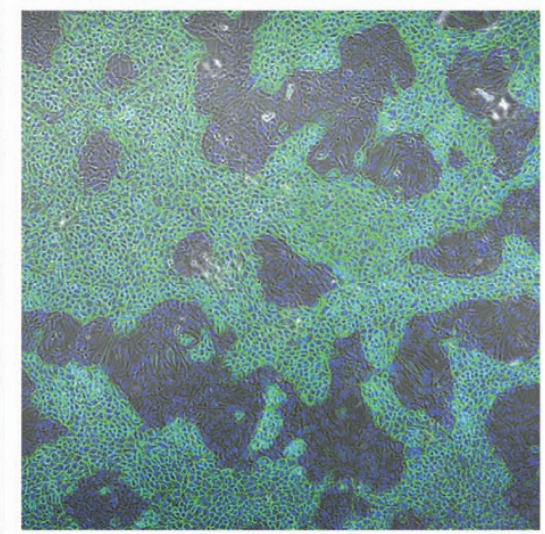

E-cadherin/ Hoechst b

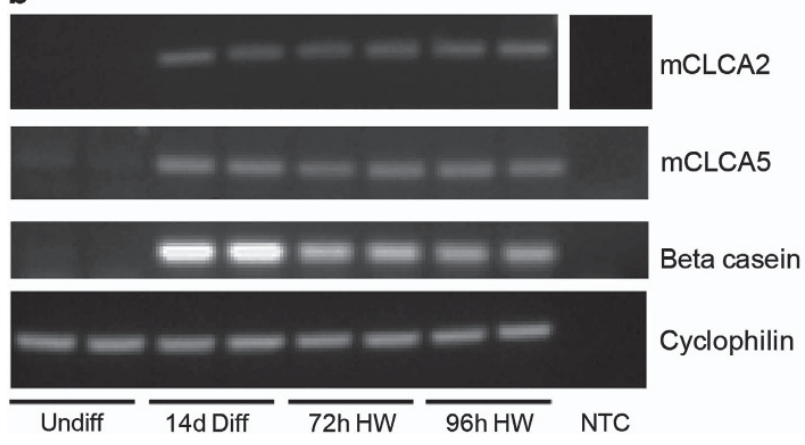

c

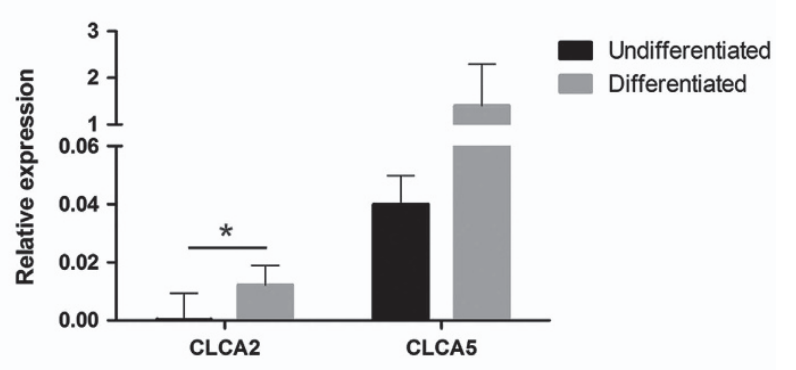

Figure 1 KIM-2 mammary epithelial cells exhibit upregulation of mCLCA2 and mCLCA5 expression upon differentiation, which persists during hormone withdrawal. (a) Upon stimulation with prolactin, KIM-2 cells form dome-like epithelial islands (d), which exhibit robust E-cadherin expression, surrounded by more elongate, loosely arranged cells (I). Immunofluorescence staining for E-cadherin (green) and DNA (Hoechst; blue). Right panel shows merged images. Scale bar $=100 \mu \mathrm{m}$. (b) RT-PCR analysis for mCLCA2, mCLCA5, $\beta$-casein and cyclophilin A (housekeeping gene), in KIM-2 cells that are undifferentiated (undiff), KIM-2 cells that have received lactogenic hormones for 14 days (14d Diff), or KIM-2 cells that have received lactogenic hormones for 11-14 days and have then undergone hormone withdrawal for 72 (72 h HW) or $96 \mathrm{~h}$ (96 h HW). Two independent biological repeats of each condition are shown. (c) Expression of mCLCA2 and mCLCA5 in undifferentiated and differentiated KIM-2 cells, measured by qRT-PCR relative to expression of cyclophilin A (housekeeping gene); values are mean \pm S.D. from at least three biological repeats; ${ }^{*} P<0.05$, as determined by Student's $t$-test. NTC, no template control

mammary tumour cell lines, including $4 \mathrm{~T} 1$ cells, where reexpression inhibits proliferation. ${ }^{22}$ Thus, hCLCA2 and its murine orthologue have been proposed as tumour suppressor genes in breast cancer. ${ }^{23,24}$ However, other investigators have demonstrated an upregulation of hCLCA2 in triple-negative breast cancer patients with a poor prognosis, ${ }^{25}$ perhaps suggesting breast cancer subtype specific roles, and exemplifying the need for further characterization of this poorly understood protein family.

Other murine genes include $\mathrm{mClca} 1$ and $\mathrm{mClca} 2$ that share $\sim 96 \%$ complementary DNA sequence identity, and encode mCLCA1 and mCLCA2, respectively. ${ }^{26}$ These proteins are reciprocally expressed during mammary gland development with $\mathrm{mCLCA} 2$ being expressed at high levels during lactation and involution, whereas mCLCA1 expression is suppressed during involution. ${ }^{27}$ This pattern suggests different functional roles for these highly related genes.

Given the pro-tumourigenic potential of the involution mammary microenvironment, the critical role of Stat3 in orchestrating regression and the intriguing regulation of mCLCA proteins, we hypothesized that mammary epithelial expression of Stat3 would influence the expression of mCLCA proteins.

\section{Results}

Expression of mCLCA2 and mCLCA5 in mammary epithelial KIM-2 cells is modulated by Stat 3 activity. To interrogate the hypothesis that mammary epithelial expression of Stat3 will influence the expression of MCLCA proteins, we first wished to establish baseline levels of expression of mCLCA2 and mCLCA5 in the KIM-2 conditionally immortal mouse mammary epithelial cell line. KIM-2 cells were maintained in an undifferentiated state and were subsequently differentiated by addition of lactogenic hormones, resulting in the emergence of two phenotypically distinct populations - an epithelial component expressing E-cadherin, and forming distinct islands and dome-like structures, and a more loosely arranged population of elongated cells around these islands (Figure 1a), as previously described. ${ }^{28}$ Differentiation was confirmed by robust upregulation in expression of the milk protein $\beta$-casein (Figure 1b). 
Interestingly, both mCLCA2 and mCLCA5 exhibited a pronounced upregulation in expression upon differentiation, with mCLCA5 exhibiting higher levels of expression than mCLCA2. This elevated expression persisted during the first $96 \mathrm{~h}$ of hormone withdrawal, a situation that mimicks involution (Figure 1).

Given the striking upregulation of mCLCA2 and mCLCA5 upon differentiation of the KIM-2 cells and during the involution-like process precipitated by hormone withdrawal, we wished to investigate the impact of Stat3 signalling. Undifferentiated KIM-2 mammary epithelial cells were stimulated with either LIF or OSM to activate Stat3 by phosphorylation. Basal levels of expression of mCLCA2 and mCLCA5 were low/undetectable in undifferentiated KIM-2 cells (Figures 1b, 2a and b), but a modest increase in expression of mCLCA2 was seen upon stimulation with both LIF (Figure 2a) and OSM (Figure 2b) mirroring the upregulation in phosphorylated Stat3 (pStat3). Modulation of mCLCA5 expression was not observed. As anticipated, expression of mCLCA2 was also enhanced by stimulation of differentiated $\mathrm{KIM}-2$ cells with OSM, but intriguingly this resulted in a modest decrease in mCLCA5 expression (Figure 2c), possibly reflecting differences in receptor downstream signalling pathways in differentiated cells. This was also reflected in the response of differentiated KIM-2 cells to LIF where upregulation of pStat3 was not observed (Supplementary Figure 1), despite continued expression of the LIF receptor at the RNA level (data not shown).

These findings suggest that two of the key upstream regulators of Stat3 during involution, namely LIF and OSM, regulate CLCA expression in murine mammary epithelium, possibly in a reciprocal manner, with mCLCA2 being induced, while mCLCA5 is suppressed. This result prompted us to investigate CLCA expression in vivo.

mCLCA1 and mCLCA2 are upregulated, and mCLCA5 is downregulated, during involution in a Stat3-dependent manner. Affymetrix (High Wycombe, UK) microarray data that have been previously published, ${ }^{29}$ indicated that mCLCA3 is expressed at very low levels (data not shown), whereas expression of mCLCA1 is markedly upregulated between 12 and $48 \mathrm{~h}$ of involution (first phase), and subsequently maintained from $48 \mathrm{~h}$ onwards (conventionally considered the onset of the murine irreversible second phase; Figure 3a).

To further interrogate a possible relationship between Stat3 activity and CLCA protein expression, we utilized mice with a mammary epithelial cell-specific conditional deletion of Stat3 (Stat3 ${ }^{f / / f l}$; BLG-Cre; hereafter referred to as Stat3 KO) and compared these with age-matched controls lacking Cre expression (control). Using qRT-PCR, we compared expression of mCLCA1, mCLCA2 and mCLCA5 during lactation and involution, utilizing samples collected at $42 \mathrm{~h}$ involution, close to the onset of the irreversible phase (unpublished data).

mCLCA1, mCLCA2 and mCLCA5 are all significantly upregulated during involution, particularly, at the onset of the second phase. Strikingly, expression of mCLCA1 and mCLCA2 is almost entirely abrogated in the absence of epithelial Stat3 (Figures 3b and c). Conversely, mCLCA5 expression exhibits a different pattern of expression reaching maximal levels at the $42 \mathrm{~h}$ of involution 'switch point' to irreversibility, and thereafter declining. Interestingly, expression is not abrogated by deletion of Stat3, and is instead significantly upregulated in the Stat3 $\mathrm{KO}$ mice at 24, 72 and
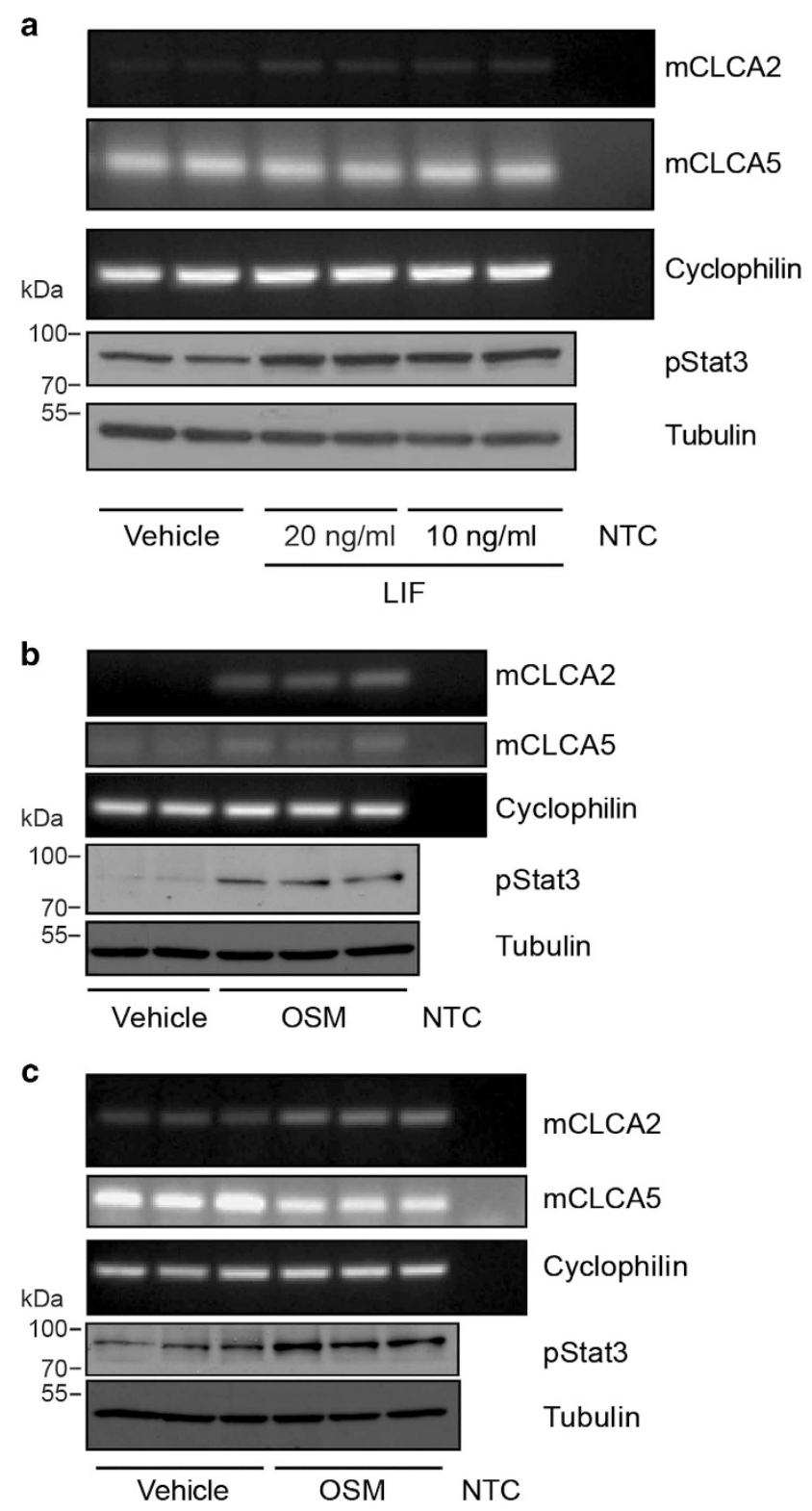

Figure 2 Undifferentiated mammary epithelial KIM-2 cells exhibit low levels of expression of mCLCA2 and mCLCA5, and expression of mCLCA2 may be upregulated upon stimulation with LIF or OSM. Differentiated KIM-2 cells upregulate expression of $\mathrm{mCLCA} 2$ upon stimulation with OSM and downregulate expression of mCLCA5. (a) RT-PCR analysis for mCLCA2, mCLCA5 and cyclophilin A (housekeeping gene), and western blot analysis of phosphorylated Stat3 (pStat3) and tubulin expression in undifferentiated KIM-2 cells stimulated with either vehicle or 10 or $20 \mathrm{ng} / \mathrm{ml} \mathrm{LIF}$. Two biological repeats are shown. (b) RT-PCR analysis for mCLCA2, mCLCA5 and cyclophilin A, and western blot analysis of pStat3 and tubulin expression in undifferentiated KIM-2 cells stimulated with either vehicle or $25 \mathrm{ng} / \mathrm{ml}$ OSM. Two (vehicle) or three (OSM stimulated) biological repeats are shown. (c) RTPCR analysis for mCLCA2, mCLCA5 and cyclophilin A, and western blot analysis of pStat3 and tubulin expression in differentiated KIM-2 cells stimulated with either vehicle or $25 \mathrm{ng} / \mathrm{ml}$ OSM. Three biological repeats are shown. NTC, no template control 
a

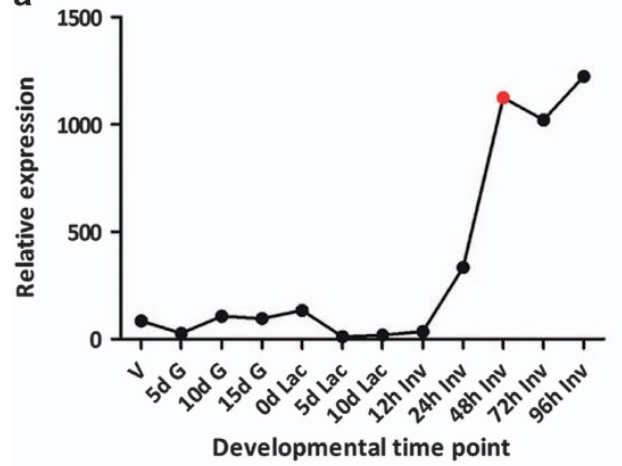

C

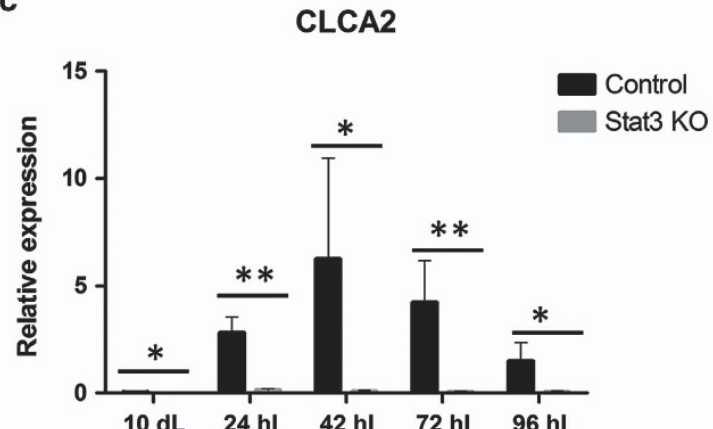

b

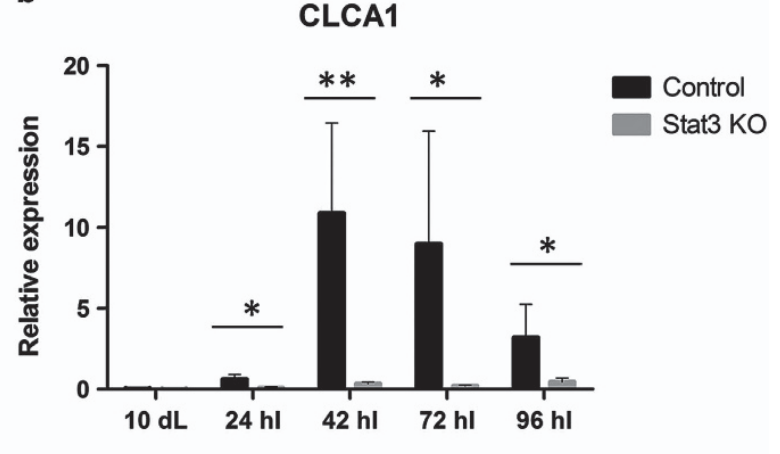

d

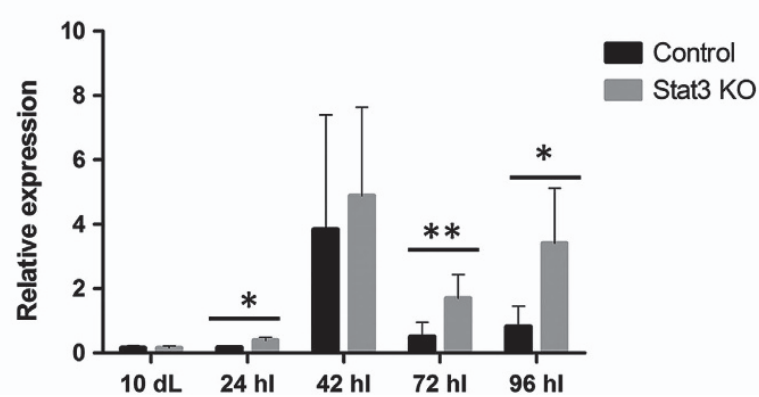

Figure $3 \mathrm{mCLCA} 1$ and $\mathrm{mCLCA} 2$ are profoundly upregulated during involution in a Stat3-dependent manner. By contrast, $\mathrm{mCLCA} 5$ is upregulated in the absence of epithelial Stat3 signalling. (a) Microarray expression profile for MCLCA1 derived from 12 different time points in the developmental cycle of the mouse mammary gland. Expression of mCLCA1 (b), mCLCA2 (c) and mCLCA5 (d) in control and Stat3 KO mice measured by qRT-PCR relative to expression of cyclophilin A (housekeeping gene). Values are mean \pm S.D. from at least three biological repeats; ${ }^{*} P<0.05,{ }^{* \star} P<0.01$ as determined by Student's $t$-test. $d \mathrm{G}$, days gestation; dL, days lactation; $\mathrm{d}$ Lac, days lactation; hl, hours involution; h Inv, hours involution; V, virgin

$96 \mathrm{~h}$ of involution (Figure $3 \mathrm{~d}$ ). This correlates with the observation that MCLCA5 is downregulated upon stimulation of Stat3 activity in OSM-stimulated differentiated KIM-2 cells (Figure 2c).

At $96 \mathrm{~h}$ of involution, modest expression of mCLCA5 is detectable in mammary epithelial cells in both control and Stat3 KO mice (Figure 4a and Supplementary Figure 2A). In control animals, expression of $\mathrm{mCLCA} 5$ is multifocally variable in its intensity, which may reflect differing levels of mammary alveolar regression within the gland at this time (Figure $4 \mathrm{~b}$ ). Correlating with the qRT-PCR data (Figure 3d), there is apparent increased expression of mCLCA5 in the absence of epithelial Stat3 signalling (Figure $4 \mathrm{~b}$ and Supplementary Figure $2 \mathrm{~B}$ ). Intriguingly, mCLCA5 is present in some intraluminal shed (dying) epithelial cells, although this is not a uniform finding.

mCLCA5 and Stat3 may be reciprocally expressed during tumourigenesis. We considered our observations in the Stat3 KO mice to be highly relevant, given that constitutively active Stat3 signalling is observed in approximately half of primary breast cancers ${ }^{30}$ and that mCLCA5 is the murine orthologue of the human putative tumour suppressor hCLCA2, as previously discussed. ${ }^{20}$ It therefore appeared logical that Stat3 signalling in tumours may have an inhibitory effect upon expression of mCLCA5 and thus we explored this hypothesis in the 4T1 murine syngeneic tumour model, which has previously been reported to exhibit minimal expression of mCLCA5. ${ }^{22}$

Implantation of $4 \mathrm{~T} 1$ cells into the mammary fat pad of syngeneic mice resulted in the development of fast growing tumours comprising pleomorphic cells with a high mitotic rate as previously described ${ }^{31,32}$ (Figures 5a-c). pStat3 was upregulated in tumours derived from implantation of 4T1 cells, compared with 4T1 cells maintained in vitro, in which pStat3 levels are low. Unexpectedly, we discovered that a higher level of pStat3 accompanied a higher level of mCLCA5 expression (Figure 5d). Interestingly, the majority of nuclear pStat3 expression was seen at the invasive edge of the tumour, where cells with morphology consistent with tumour cells, fibroblasts and immune cells, all exhibited nuclear pStat3 localization (Figure $6 a) .{ }^{33}$ The subgross pattern of localization of mCLCA5 was strikingly similar: punctate cytoplasmic mCLCA5 expression was present predominantly at the edge of the mass (Figure 6b). Granular or punctate cytoplasmic staining could be consistent with the expression of a protein within intracellular organelles - potentially a secretory product. $^{15}$

The tumours resulting from implantation of the 4T1 cells were morphologically heterogeneous, with abundant expression of vimentin (Supplementary Figure 3). Cells expressing E-cadherin and vimentin were admixed at the invasive front, with multifocal apparent co-expression (Figure $7 \mathrm{a}$ and Supplementary Figure 4A). This observation underlines the 

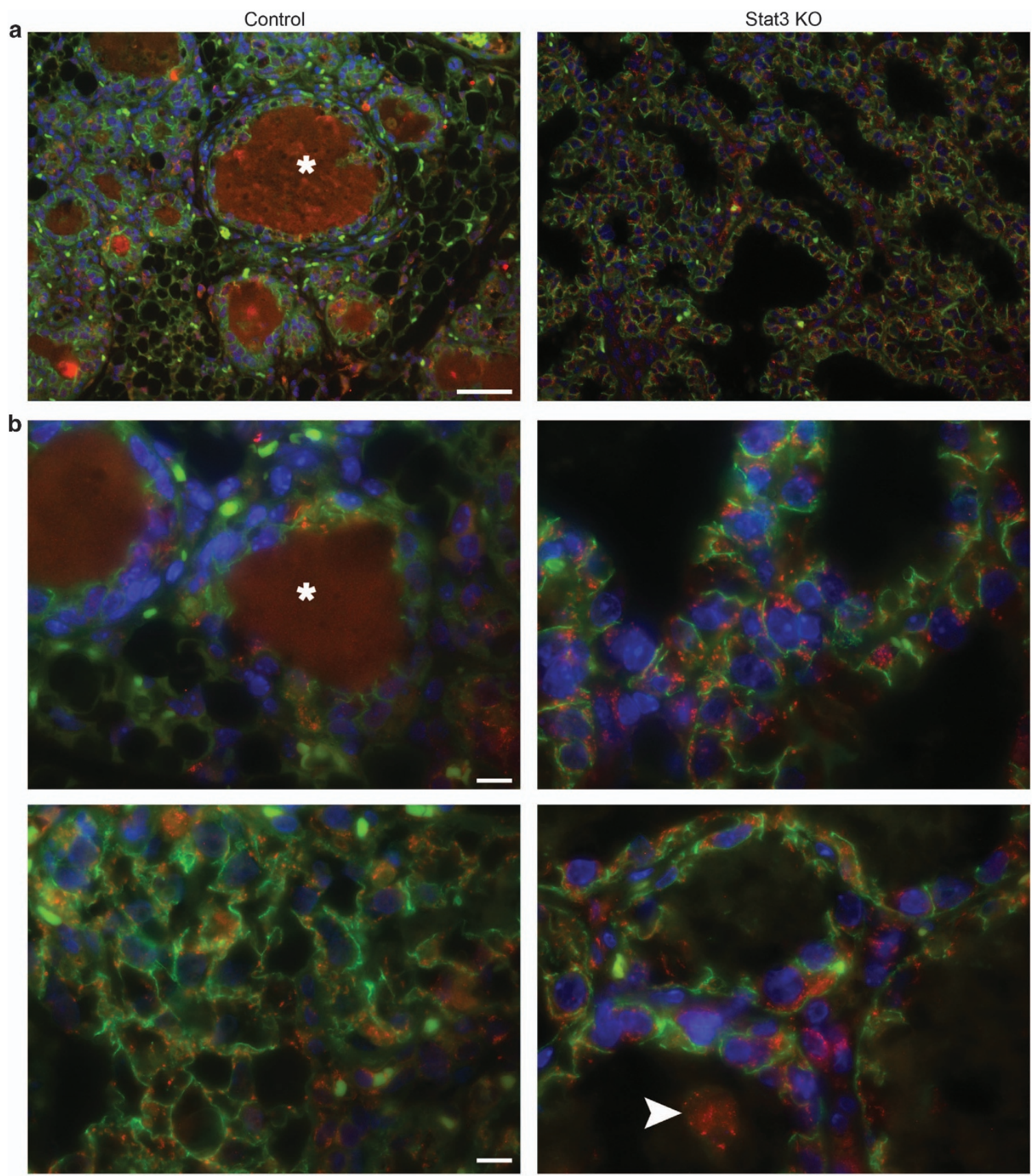

mCLCA5/ E-cadherin/ Hoechst

Figure $4 \mathrm{mCLCA5}$ protein is expressed during mammary gland involution and is subjectively more abundant in the absence of epithelial Stat3 signalling. Immunofluorescence staining for mCLCA5 (red) and E-cadherin (green), and DNA (Hoechst; blue) on control and Stat3 KO tissue at $96 \mathrm{~h}$ of involution. Scale bar $=50 \mu \mathrm{m}$ (a) and $10 \mu \mathrm{m}$ (b). Staining of residual milk (*) was considered non-specific. Some, but not all shed cells exhibited positive staining for mCLCA5 (arrowhead). Representative images are displayed. Sections from five mice were examined (control $n=2$; Stat3 $\mathrm{KO} n=3$ )

potential importance of localization of mCLCA5 to the invasive front, where cells may be assuming more mesenchymal characteristics. As $4 \mathrm{~T} 1$ cells have been previously demonstrated to express modest amounts of vimentin and robust levels of E-cadherin in vitro, ${ }^{34}$ expression of both proteins in vivo was perhaps unsurprising, given the aggressive phenotype of the tumours. Although the cellular heterogeneity and apparent co-expression of vimentin and E-cadherin by the 4T1 cells precluded definitive identification, by co-staining, of the mCLCA5 expressing cells as 4T1 cells, we identified E-cadherin and mCLCA5 dual-positive cells that we considered likely to be $4 \mathrm{~T} 1$ cells (Figure $7 \mathrm{~b}$ and Supplementary Figure 4B).

Using immunofluorescence to further characterize the cellular populations expressing mCLCA5 and nuclear Stat3 (as a surrogate read-out of Stat3 activity), we identified at least two different cell populations at the edge of the tumour 
a

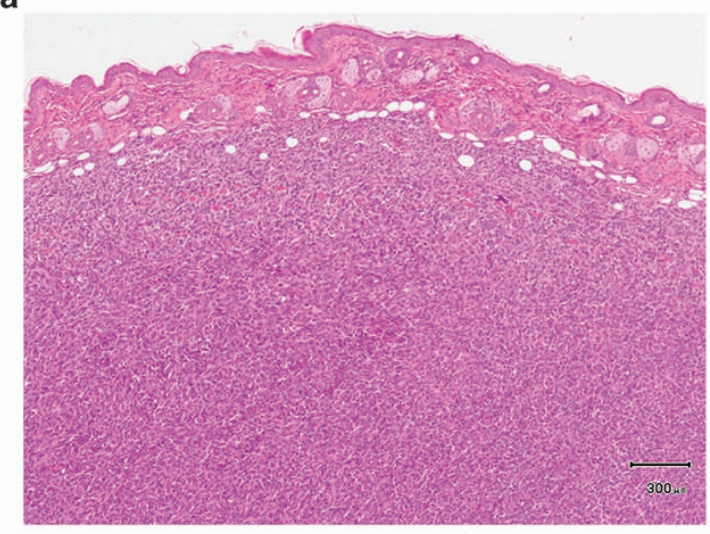

C

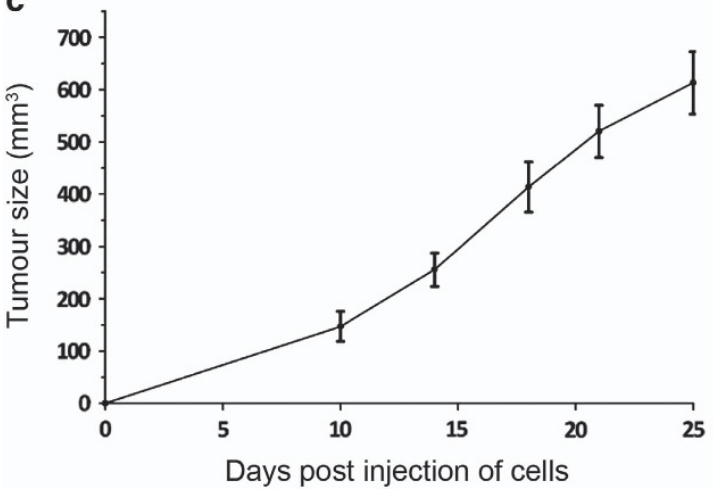

b

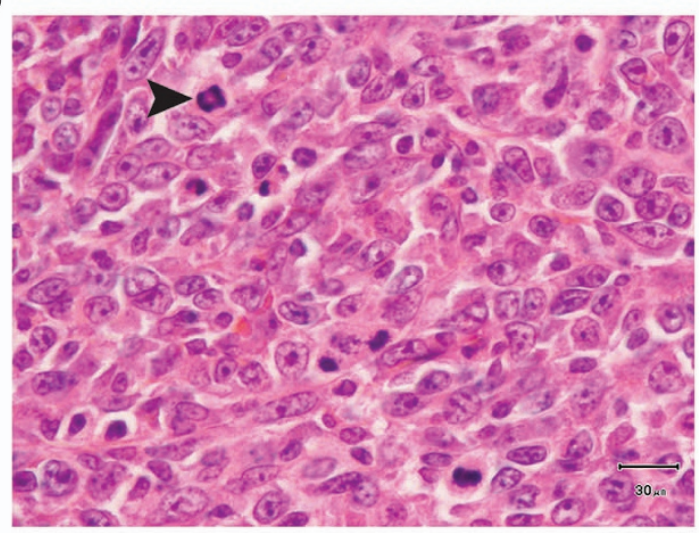

d

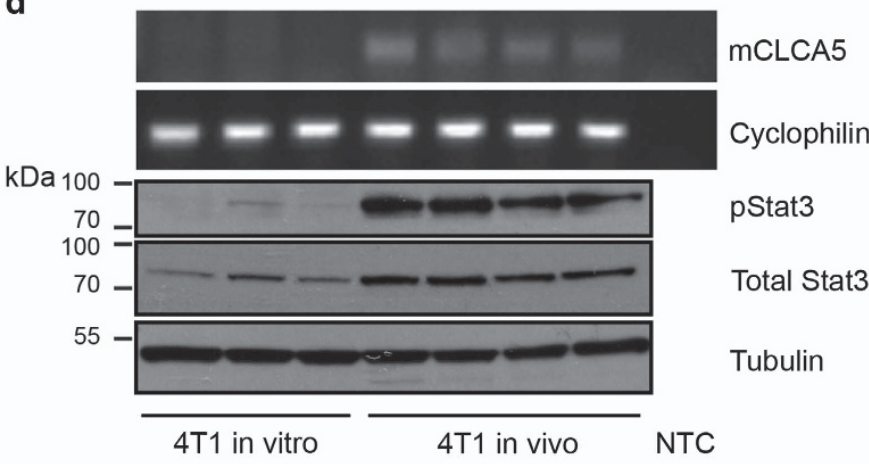

Figure 5 mCLCA5 is expressed in tumours resulting from implantation of 4T1 cells. (a and $\mathbf{b}$ ) Representative photomicrographs demonstrating that orthotopic tumours derived from 4T1 cells are densely cellular, with minimal stroma and a moderately to markedly pleomorphic phenotype with numerous mitoses. Haematoxylin and eosin stain. Scale bar $=300 \mu \mathrm{m}$ (a) and $30 \mu \mathrm{m}$ (b). Arrowhead indicates a mitotic figure. Several mitoses are present in this field. (c) Average tumour size in $\mathrm{mm}^{3}$ for tumours derived from injection of $1 \times 10^{5} 4 \mathrm{~T} 1$ cells in the mammary fat pad. Values are mean \pm S.D. from four biological repeats. (d) RT-PCR analysis for mCLCA5 and cyclophilin A, and western blot analysis of phosphorylated Stat3 (pStat3), total Stat3 and tubulin expression in 4T1 cells maintained in culture (4T1 in vitro) and in tumours resulting from implantation of 4T1 cells into the mammary fat pad of syngeneic mice (4T1 in vivo). Three independent biological repeats are shown for 4T1 in vitro; for 4T1 implanted in mice, four tumours from separate individuals are represented. NTC, no template control

(Figure 7c and Supplementary Figure 4C). Strikingly, the majority of cells either exhibited intense nuclear staining for Stat3 or strong punctate cytoplasmic staining for mCLCA5, but very few cells were identified that co-expressed mCLCA5 in the presence of nuclear Stat3. Although some of the cells expressing Stat3 exhibited morphology consistent with stromal cells or tumour-associated immune cells, others were consistent with neoplastic cells (Figure 7c).

We therefore suggest that in individual neoplastic cells, the negative regulation of $\mathrm{mCLCA} 5$ by $\mathrm{pStat} 3$ remains functional. Although both mCLCA5 and pStat3 are expressed predominantly at the invasive edge of the tumour, minimal colocalization of nuclear Stat3 and cytoplasmic mCLCA5 is observed. It seems likely that mCLCA5 expression may be predominantly in the tumour cells, whereas pStat3 nuclear localization is seen in both the tumour cells and immune and stromal compartments at the invasive front. Thus, activity of pStat3, a known breast cancer oncogene, ${ }^{30,35}$ is likely to be critical to the invasive nature of the neoplastic cells at the tumour margin.

Other investigators have suggested that CLCA proteins may act as extracellular signalling molecules ${ }^{17,36}$ and it is tempting to speculatively attribute such as role to mCLCA5 in this context at the invasive edge of the tumour (Figure 7).

\section{Discussion}

We have demonstrated that mCLCA1 and mCLCA2 are profoundly downregulated in the absence of mammary epithelial Stat3 signalling during post-lactational regression, suggesting a close direct or indirect relationship between Stat3 activity and expression of mCLCA1 and mCLCA2. This exciting finding warrants further investigation in the context of the function of these proteins within the mammary gland.

Furthermore, our data suggest that during mammary gland regression, mCLCA5 expression is partially suppressed by epithelial Stat3 activity. Given the pro-tumourigenic nature of the involution mammary microenvironment, this is an important finding. However, data from orthotopic tumours derived from implantation of $4 \mathrm{~T} 1$ cells into mice suggest that the relationship between mCLCA5 and Stat3 activity may be complex, with separate populations of cells expressing high levels of mCLCA5 or transcriptionally active (nuclear) Stat3. This suggests that Stat3 retains a suppressive effect on 
a
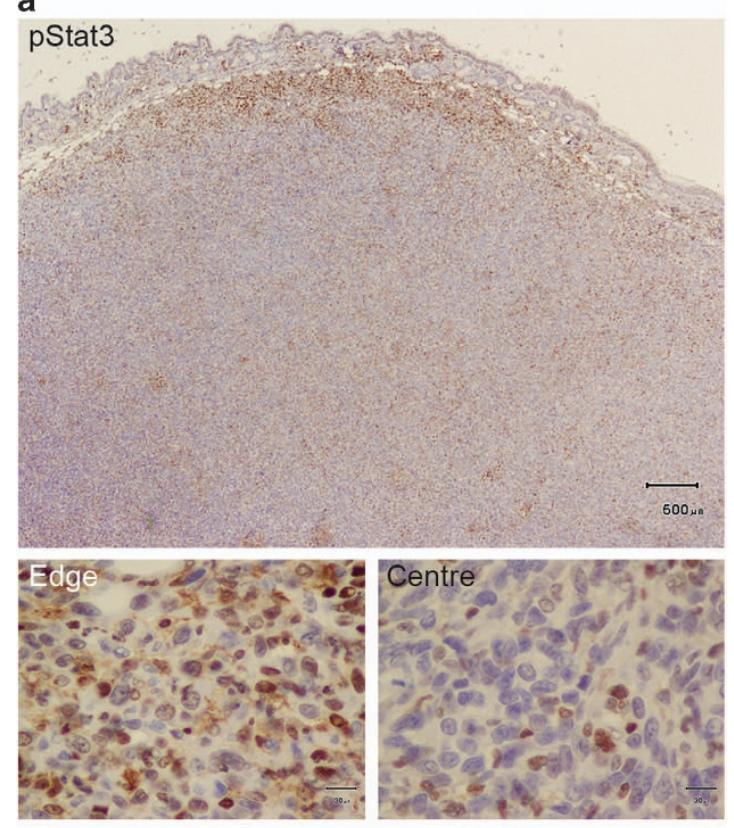

b
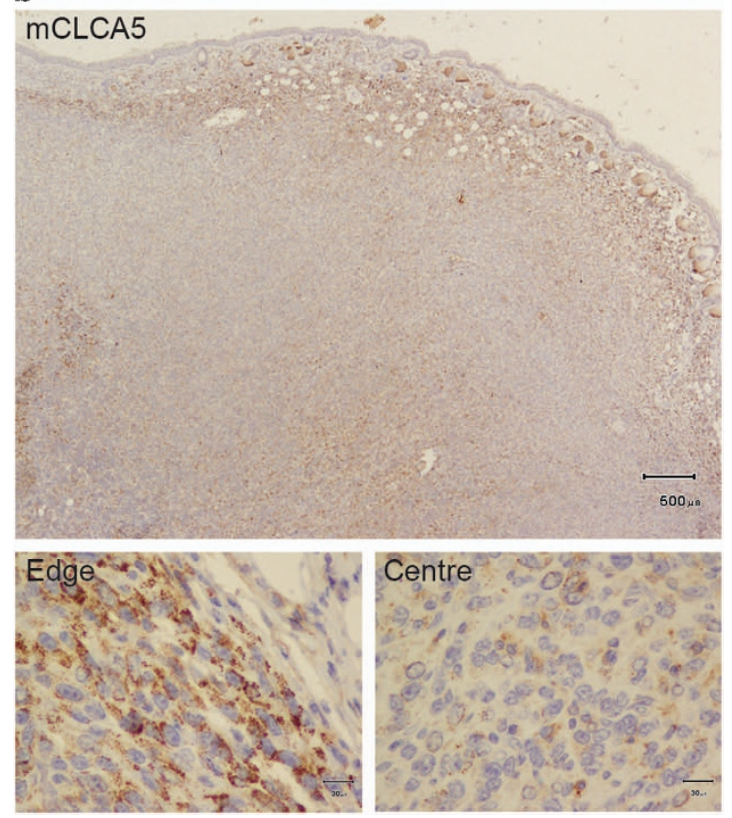

Figure 6 Distinct cell populations express mCLCA5 and nuclear Stat3 at the invasive edge of tumours resulting from implantation of $4 \mathrm{~T} 1$ cells. (a and $\mathbf{b}$ ) Immunohistochemical staining for pStat3 (a) and MCLCA5 (b) on adjacent sections of orthotopic tumours derived from implantation of $4 \mathrm{~T} 1 \mathrm{cellls}$. Haematoxylin counterstain. Scale bar $=500 \mu \mathrm{m}$. The micrographs at higher magnification show representative staining from the edge and centre of the mass as indicated. Scale bar $=30 \mu \mathrm{m}$

mCLCA5 expression in 4T1 tumour cells. It is in accordance with the findings of others, and therefore tempting to speculate, that downregulation of MCLCA5 in the presence of cells expressing Stat3 activity reflects an invasive phenotype in these cells and potential epithelial-mesenchymal transition. ${ }^{16,22,24}$

However, our data also raise the question of the role of the observed mCLCA5 expression at the invasive front. One interpretation is that $\mathrm{mCLCA} 5$ expression at the invasive edge may be beneficial to tumour growth with mCLCA5 acting in a non-cell autonomous manner to augment tumour cell proliferation. ${ }^{37}$ However, in this context it is important to note the compelling data demonstrating that re-expression of mCLCA5 in 4T1 cells inhibits proliferation ${ }^{22}$ and that loss of hCLCA2 expression in human MCF10A cells augments proliferation of the cells, ${ }^{24}$ whereas re-expression elicits a reduction in tumour cell growth. ${ }^{19}$ In the in vivo 4T1 murine model described in our study, cellular behaviour may be influenced by the presence of subpopulations of neoplastic cells in which either Stat3 activity suppresses mCLCA5 expression, or mCLCA5 is expressed in the absence of Stat3 activity. The influence of the immune cell compartment also needs to be considered particularly given that the 4T1 model involves introduction of syngeneic tumour cells into immunecompetent mice. It is possible that mCLCA5 secretion is stimulated in host cells, such as stromal components or immune cells, in an attempt to negatively regulate tumour growth. Currently, the role of mCLCA5 at the invasive front of the tumour is unclear. Future work will require development of inducible models of mCLCA5 expression and/or Stat3 activity in vivo in different cell types to understand the relationship between the key oncogene Stat3 and the intriguing factor mCLCA5.

Although in normal mammary gland, Stat3 regulates cell death, breast cancer cells frequently become addicted to Stat3 and require Stat3 activity for survival. Our data demonstrate that Stat3 is a negative regulator of mCLCA5 during mammary gland involution, and that in tumourigenesis in an immunecompetent murine model, mCLCA5 expression is suppressed in cells with high levels of nuclear Stat3. Stat3 appears to be a cell autonomous negative regulator of this protein.

\begin{abstract}
Materials and Methods
Cell culture. Maintenance and differentiation of KIM-2 cells and instigation of hormone withdrawal, were as previously described. ${ }^{28}$ Human LIF (a generous gift from $\mathrm{Dr}$ Jennifer Nichols) or recombinant mouse OSM (495-MO, R\&D Systems, Abingdon, UK) were used at a final concentration of 10 or $20 \mathrm{ng} / \mathrm{ml}$ (LIF), or $25 \mathrm{ng} /$ $\mathrm{ml}$ (OSM). For imaging, KIM-2 cells were differentiated in $35 \mathrm{~mm}$ diameter glass bottom dishes (81158, ibidi, Planegg, Martinsried, Germany). Murine 4T1 cells were purchased from ATCC (Teddington, Middlesex, UK) and were maintained according to ATCC protocols.
\end{abstract}

RT-PCR and qRT-PCR. Standard protocols were followed. ${ }^{38}$ The primer sequences utilized are detailed in Supplementary Table 1.

Immunoblotting. Standard protocols were followed. ${ }^{38}$ Antibodies employed were: anti-phospho-Stat3 (1:1000; Tyr705: \#9131), anti-Stat3 (1:1000; \#12640), antiGAPDH (1:5000; \#5174) (all Cell Signaling Technology, Danvers, MA, USA), and anti-alpha-tubulin (1:5000; AB6160, Abcam, Cambridge, UK).

Animal husbandry. Stat ${ }^{f l / f i} ;$ BLG-Cre mice ${ }^{39,40}$ were utilized and involution induced as previously described. ${ }^{9}$ All animals were treated according to the local ethical committee and the UK Home Office guidelines.

4T1 tumour model. A final volume of $16 \mu$ l containing $1 \times 10^{5} 4 \mathrm{~T} 1$ cells with matrigel (basement membrane matrix growth factor reduced; BD 354230; BD Biosciences, Erembodegem, Belgium) was injected into the right- or left-fourth mammary gland fat pad of $\sim 12$-week-old virgin Balb/c mice under anaesthesia and analgesia. ${ }^{41}$ Tumour development was monitored, mice were killed and tumours were harvested at day 25 post injection. Tumour tissue (lacking normal tissue margins) was collected for RNA and protein extraction. Tissue for histological 
a

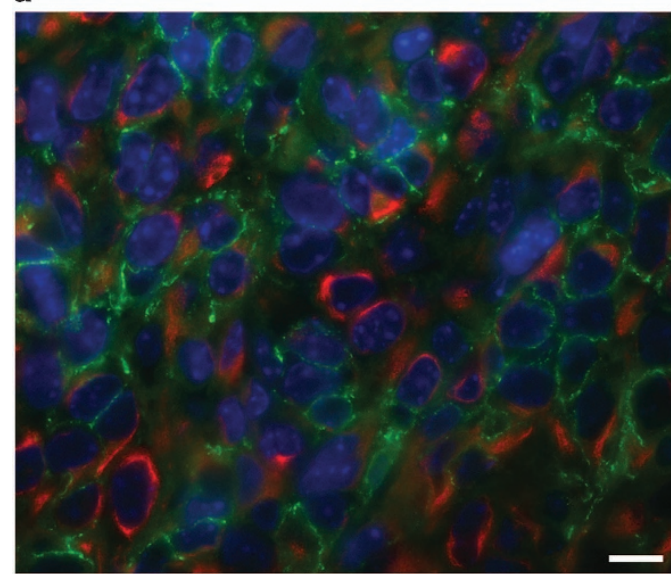

Vimentin/ E-cadherin/ Hoechst

c

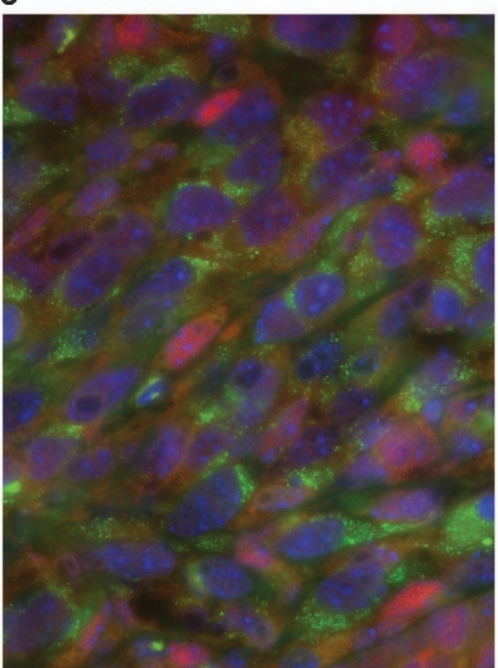

Stat3/ mCLCA5/ Hoechst b

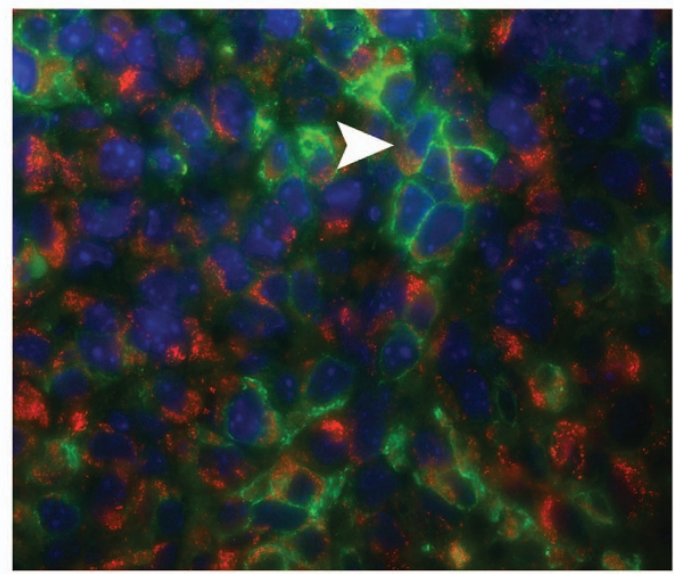

mCLCA5/ E-cadherin/ Hoechst d

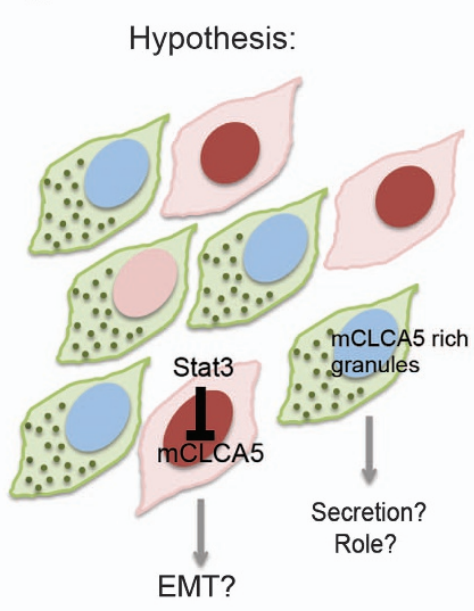

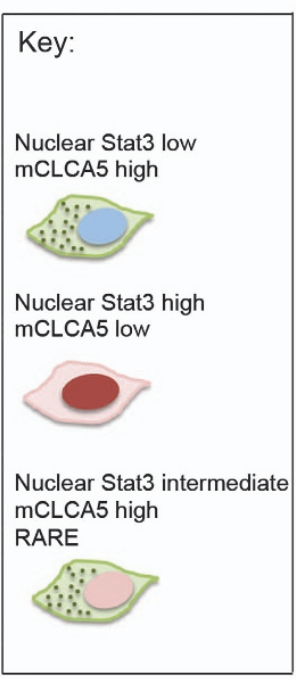

Figure 7 Although both mCLCA5 and pStat3 are expressed predominantly at the invasive edge of the tumour, minimal co-localization of nuclear Stat3 and cytoplasmic mCLCA5 is observed. Representative images showing the edge of orthotopic tumours derived from implantation of $4 \mathrm{~T} 1 \mathrm{cells}$. (a) Immunofluorescence staining for vimentin (red), E-cadherin (green) and DNA (Hoechst; blue). Scale bar $=10 \mu \mathrm{m}$. (b) Immunofluorescence staining for mCLCA5 (red), E-cadherin (green) and DNA (Hoechst; blue). Groupings of E-cadherin-positive cells express mCLCA5 (arrowhead). (c) Immunofluorescence staining for Stat3 (red), mCLCA5 (green) and DNA (Hoechst; blue). In each case, images are representative of results from three separate mice and are captured at the same magnification. (d) Graphical summary of hypothesis that subpopulations of tumour cells may express high levels of the oncogene Stat3 or mCLCA5, and that MCLCA5 may be secreted

sectioning was dissected. Formalin fixation, histological sectioning and staining followed routine protocols.

Immunostaining. KIM-2 cells in glass bottom dishes were fixed for 10 min in ice cold methanol and immunofluorescence staining for E-cadherin (1:400; \#3195; Cell Signaling Technology) was carried out following a standard protocol.

Paraffin embedded tumour specimens were prepared as three micron sections on positively charged slides (Snowcoat; Surgipath Europe Ltd, Peterborough, UK). Immunohistochemical staining for mCLCA5 (1:500; sc99224; Santa Cruz Biotechnology, Dallas, TX, USA), vimentin (1:500; \#5741) and murine pStat3 (1:100; Tyr705: \#9145) (both Cell Signaling Technology) was carried out using a routine protocol employing an automated immunohistochemistry system (Dako Autostainer; Dako, Ely, UK).

Immunofluoresence staining for E-cadherin (1:500; 610182, BD Biosciences, Oxford Science Park, Oxford, UK), mCLCA5 (1:100; sc99224; Santa Cruz Biotechnology), vimentin (1:200; \#5741) and murine total Stat3 (1:100; \#9139) (both Cell Signaling Technology, Danvers, MA, USA) was carried out following de-paraffinization and antigen retrieval for $20 \mathrm{~min}$ at $90^{\circ} \mathrm{C}$ using Dako Envision
Flex Target Antigen Retrieval Solution High pH in a PT Link and Pre-Treatment Module for Tissue Specimens (both Dako). For Fc receptor blocking prior to staining for murine total Stat3 (murine monoclonal antibody), anti-mouse CD16/CD32 clone 93 was utilized (1:500; 14-0161-85, eBioscience Ltd, Cheshire, UK). Isotype- and species-matched immunoglobulins were used as a negative control.

\section{Conflict of Interest}

The authors declare no conflict of interest.

Acknowledgements. KH was funded by the Medical Research Council (MRC) (project grant number G0900980) and is a co-applicant on MRC project grant number MR/K011014/1. MB was funded by a Department of Pathology PhD studentship. SP was funded by the MRC (project grant number MR/K011014/1). Work in CJW's laboratory is funded by the MRC, NC3Rs and the Wellcome Trust. We thank Dr Jennifer Nichols, University of Cambridge, for providing the LIF for KIM-2 cell stimulation, and Helen Skelton, Madeline Fordham and Scott Dillon for preparation of tissue sections and automated immunohistochemistry. The data detailed in this 
manuscript were presented at the 2015 ACVP Annual Meeting, Minneapolis, USA, 17-21 October 2015 (presentation: 18 October 2015). We thank the members of the Watson laboratory for their ongoing support and helpful discussion.

\section{Author contributions}

$\mathrm{KH}$ carried out majority of the experiments; MB carried out the experiments for Supplementary Figure 1 and performed cell culture; SP assisted in surgical implantation of 4T1 cells; $\mathrm{KH}$ and CJW analyzed the data and wrote the paper. All authors have approved the final submitted version.

\section{Ethical Background Statement}

All animals were treated according to the local ethical committee and the UK Home Office guidelines.

1. Nguyen AV, Pollard JW. Transforming growth factor beta3 induces cell death during the first stage of mammary gland involution. Development 2000; 127: 3107-3118.

2. Kritikou EA, Sharkey A, Abell K, Came PJ, Anderson E, Clarkson RW et al. A dual, non-redundant, role for LIF as a regulator of development and STAT3-mediated cell death in mammary gland. Development 2003; 130: 3459-3468

3. Schere-Levy C, Buggiano V, Quaglino A, Gattelli A, Cirio MC, Piazzon I et al. Leukemia inhibitory factor induces apoptosis of the mammary epithelial cells and participates in mouse mammary gland involution. Exp Cell Res 2003; 282: 35-47.

4. Tiffen PG, Omidvar N, Marquez-Almuina N, Croston D, Watson CJ, Clarkson RW. A dual role for oncostatin $\mathrm{M}$ signaling in the differentiation and death of mammary epithelial cells in vivo. Mol Endocrinol 2008; 22: 2677-2688.

5. Hughes K, Watson CJ. The role of Stat3 in mammary gland involution: cell death regulator and modulator of inflammation. Horm Mol Biol Clin Investig 2012; 10: 211-215.

6. Hughes $\mathrm{K}$, Watson CJ. The spectrum of STAT functions in mammary gland development. JAKSTAT 2012; 1: 151-158.

7. Chapman RS, Lourenco PC, Tonner E, Flint DJ, Selbert S, Takeda K et al. Suppression of epithelial apoptosis and delayed mammary gland involution in mice with a conditional knockout of Stat3. Genes Dev 1999; 13: 2604-2616.

8. Humphreys RC, Bierie B, Zhao L, Raz R, Levy D, Hennighausen L. Deletion of Stat3 blocks mammary gland involution and extends functional competence of the secretory epithelium in the absence of lactogenic stimuli. Endocrinology 2002; 143: 3641-3650.

9. Kreuzaler PA, Staniszewska AD, Li W, Omidvar N, Kedjouar B, Turkson J et al. Stat3 controls Iysosomal-mediated cell death in vivo. Nat Cell Biol 2011; 13: 303-309.

10. Sargeant TJ, Lloyd-Lewis B, Resemann HK, Ramos-Montoya A, Skepper J, Watson CJ. Stat3 controls cell death during mammary gland involution by regulating uptake of milk fat globules and lysosomal membrane permeabilization. Nat Cell Biol 2014; 16: 1057-1068.

11. Stein T, Morris JS, Davies CR, Weber-Hall SJ, Duffy MA, Heath VJ et al. Involution of the mouse mammary gland is associated with an immune cascade and an acute-phase response, involving LBP, CD14 and STAT3. Breast Cancer Res 2004; 6: R75-R91.

12. Lyons TR, O'Brien J, Borges VF, Conklin MW, Keely PJ, Eliceiri KW et al. Postpartum mammary gland involution drives progression of ductal carcinoma in situ through collagen and COX-2. Nat Med 2011; 17: 1109-1115

13. Takabatake Y, Oxvig C, Nagi C, Adelson K, Jaffer S, Schmidt $\mathrm{H}$ et al. Lactation opposes pappalysin-1-driven pregnancy-associated breast cancer. EMBO Mol Med 2016; 8: 388-406.

14. Callihan EB, Gao D, Jindal S, Lyons TR, Manthey E, Edgerton S et al. Postpartum diagnosis demonstrates a high risk for metastasis and merits an expanded definition of pregnancyassociated breast cancer. Breast Cancer Res Treat 2013; 138: 549-559.

15. Sala-Rabanal M, Yurtsever Z, Nichols CG, Brett TJ. Secreted CLCA1 modulates TMEM16A to activate $\mathrm{Ca}(2+)$-dependent chloride currents in human cells. Elife 2015; 4: e05875.

16. Ramena G, Yin Y, Yu Y, Walia V, Elble RC. CLCA2 interactor EVA1 is required for mammary epithelial cell differentiation. PLoS One 2016; 11: e0147489.

17. Dietert K, Mundhenk L, Erickson NA, Reppe K, Hocke AC, Kummer W et al. Murine CLCA5 is uniquely expressed in distinct niches of airway epithelial cells. Histochem Cell Biol 2015; 143: $277-287$

18. Yu Y, Walia V, Elble RC. Loss of CLCA4 promotes epithelial-to-mesenchymal transition in breast cancer cells. PLoS One 2013; 8: e83943.

19. Gruber AD, Pauli BU. Tumorigenicity of human breast cancer is associated with loss of the Ca2+-activated chloride channel CLCA2. Cancer Res 1999; 59: 5488-5491.

20. Evans SR, Thoreson WB, Beck CL. Molecular and functional analyses of two new calciumactivated chloride channel family members from mouse eye and intestine. J Biol Chem 2004; 279: 41792-41800

21. Braun J, Bothe MK, Mundhenk L, Beck CL, Gruber AD. Murine mCLCA5 is expressed in granular layer keratinocytes of stratified epithelia. Histochem Cell Biol 2010; 133: 285-299.
22. Beckley JR, Pauli BU, Elble RC. Re-expression of detachment-inducible chloride channel mCLCA5 suppresses growth of metastatic breast cancer cells. J Biol Chem 2004; 279: 41634-41641.

23. Walia V, Ding M, Kumar S, Nie D, Premkumar LS, Elble RC. hCLCA2 is a p53-inducible inhibitor of breast cancer cell proliferation. Cancer Res 2009; 69: 6624-6632.

24. Walia V, Yu Y, Cao D, Sun M, McLean JR, Hollier BG et al. Loss of breast epithelial marke hCLCA2 promotes epithelial-to-mesenchymal transition and indicates higher risk of metastasis. Oncogene 2012; 31: 2237-2246.

25. Kuo WH, Chang YY, Lai LC, Tsai MH, Hsiao CK, Chang KJ et al. Molecular characteristics and metastasis predictor genes of triple-negative breast cancer: a clinical study of triplenegative breast carcinomas. PLoS One 2012; 7: e45831.

26. Leverkoehne I, Horstmeier BA, von Samson-Himmelstjerna G, Scholte BJ, Gruber AD. Real-time RT-PCR quantitation of $\mathrm{mCLCA} 1$ and $\mathrm{mCLCA} 2$ reveals differentially regulated expression in pre- and postnatal murine tissues. Histochem Cell Biol 2002; 118 : 11-17.

27. Elble RC, Pauli BU. Tumor suppression by a proapoptotic calcium-activated chloride channel in mammary epithelium. J Biol Chem 2001; 276: 40510-40517.

28. Gordon KE, Binas B, Chapman RS, Kurian KM, Clarkson RW, Clark AJ et al. A novel cell culture model for studying differentiation and apoptosis in the mouse mammary gland. Breast Cancer Res 2000; 2: 222-235.

29. Clarkson RW, Wayland MT, Lee J, Freeman T, Watson CJ. Gene expression profiling of mammary gland development reveals putative roles for death receptors and immune mediators in post-lactational regression. Breast Cancer Res 2004; 6: R92-109.

30. Berishaj M, Gao SP, Ahmed S, Leslie K, Al-Ahmadie H, Gerald WL et al. Stat3 is tyrosinephosphorylated through the interleukin-6/glycoprotein 130/Janus kinase pathway in breast cancer. Breast Cancer Res 2007; 9: R32.

31. Heppner GH, Miller FR, Shekhar PM. Nontransgenic models of breast cancer. Breast Cancer Res 2000; 2: 331-334.

32. Aslakson CJ, Miller FR. Selective events in the metastatic process defined by analysis of the sequential dissemination of subpopulations of a mouse mammary tumor. Cancer Res 1992; 52: $1399-1405$.

33. Azare J, Doane A, Leslie K, Chang Q, Berishaj M, Nnoli J et al. Stat3 mediates expression of autotaxin in breast cancer. PLOS One 2011; 6: e27851.

34. Lou Y, Preobrazhenska O, auf dem Keller U, Sutcliffe M, Barclay L, McDonald PC et al. Epithelial-mesenchymal transition (EMT) is not sufficient for spontaneous murine breast cancer metastasis. Dev Dyn 2008; 237: 2755-2768.

35. Watson CJ, Hughes K. Breast cancer: the menacing face of Janus kinase. Cell Death Differ 2014; 21: 185-186.

36. Ching JC, Lobanova L, Loewen ME. Secreted hCLCA1 is a signaling molecule that activates airway macrophages. PLOS One 2013; 8: e83130.

37. Marusyk A, Tabassum DP, Altrock PM, Almendro V, Michor F, Polyak K. Non-cellautonomous driving of tumour growth supports sub-clonal heterogeneity. Nature 2014; 514: 54-58.

38. Hughes K, Wickenden JA, Allen JE, Watson CJ. Conditional deletion of Stat3 in mammary epithelium impairs the acute phase response and modulates immune cell numbers during post-lactational regression. J Pathol 2012; 227: 106-117.

39. Selbert S, Bentley DJ, Melton DW, Rannie D, Lourenco P, Watson CJ et al. Efficient BLGCre mediated gene deletion in the mammary gland. Transgenic Res 1998; 7: 387-396.

40. Alonzi T, Maritano D, Gorgoni B, Rizzuto G, Libert C, Poli V. Essential role of STAT3 in the control of the acute-phase response as revealed by inducible gene inactivation [correction of activation] in the liver. Mol Cell Biol 2001; 21: 1621-1632.

41. Tavera-Mendoza LE, Brown M. A less invasive method for orthotopic injection of breast cancer cells into the mouse mammary gland. Lab Anim 2016 (e-pub ahead of print 17 March 2016, doi:10.1177/0023677216640706).

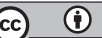

Cell Death and Disease is an open-access journal published by Nature Publishing Group. This work is licensed under a Creative Commons Attribution 4.0 International License. The images or other third party material in this article are included in the article's Creative Commons license, unless indicated otherwise in the credit line; if the material is not included under the Creative Commons license, users will need to obtain permission from the license holder to reproduce the material. To view a copy of this license, visit http://creativecommons.org/licenses/by/4.0/

(C) The Author(s) 2016 